\title{
Einkaufsdienstleister als Vermittler zwischen Krankenhaus und Industrie
}

\begin{abstract}
In Zeiten verschärfter Datenschutzrichtlinien, Zulassungsanforderungen für Medizinprodukte und einer potenziellen Gefahr durch Brexit-Auswirkungen gilt es für den Krankenhauseinkauf, permanent die Leistungsfähigkeit zu steigern und damit auch die seiner Gesundheitseinrichtung zu sichern. Bei diesen Bemühungen steht zudem die Sicherstellung einer qualitativ hochwertigen Versorgung der Patienten im Fokus des Krankenhauseinkaufs.
\end{abstract}

Es reicht schon lange nicht mehr aus, sich ausschließlich auf günstigere Einkaufskonditionen zu konzentrieren. Vielmehr liegt das große Potenzial der Häuser in einer konsequenten und nachhaltigen Verbesserung der Inhouse-Prozesse. Beispielsweise lassen sich durch die genaue Kenntnis der tatsächlich benötigten Mengen Lagerbestände signifikant verringern. Das führt zu einer Ressourcenoptimierung sowohl im Krankenhaus als auch beim Medizinproduktehersteller und reduziert gleichzeitig den Materialverfall.

Durch die Digitalisierung wiederkehrender Arbeitsabläufe, nicht nur in der Materialbeschaffung und Lagerverwaltung, sondern auch im Zuge der Patientenaufnahme sowie in den Operationssälen, werden die Mitarbeiter in der Pflege und in der Verwaltung nachhaltig entlastet. Das ermöglicht Krankenhäusern, eigenständig auf die vor allem im Gesundheitsbereich weiter zunehmende Personalknappheit zu reagieren. Politische Initiativen wie beispielsweise das Pflegepersonalstärkungsgesetz sollen die Bemühungen der Krankenhäuser und Gesundheitseinrichtungen zusätzlich unterstützen. Ob das Gesetz den erhofften Erfolg bringen wird, bleibt abzuwarten.

Doch auch auf die Wirtschaftlichkeit eines Krankenhauses hat die Digitalisierung einen großen Einfluss. Nur wenn ein Krankenhaus dazu in der Lage ist, die im Zuge der Patientenbehandlung erbrachten Leistungen gegenüber den Kostenträgern auch vollumfänglich abzurechnen sowie die ver-

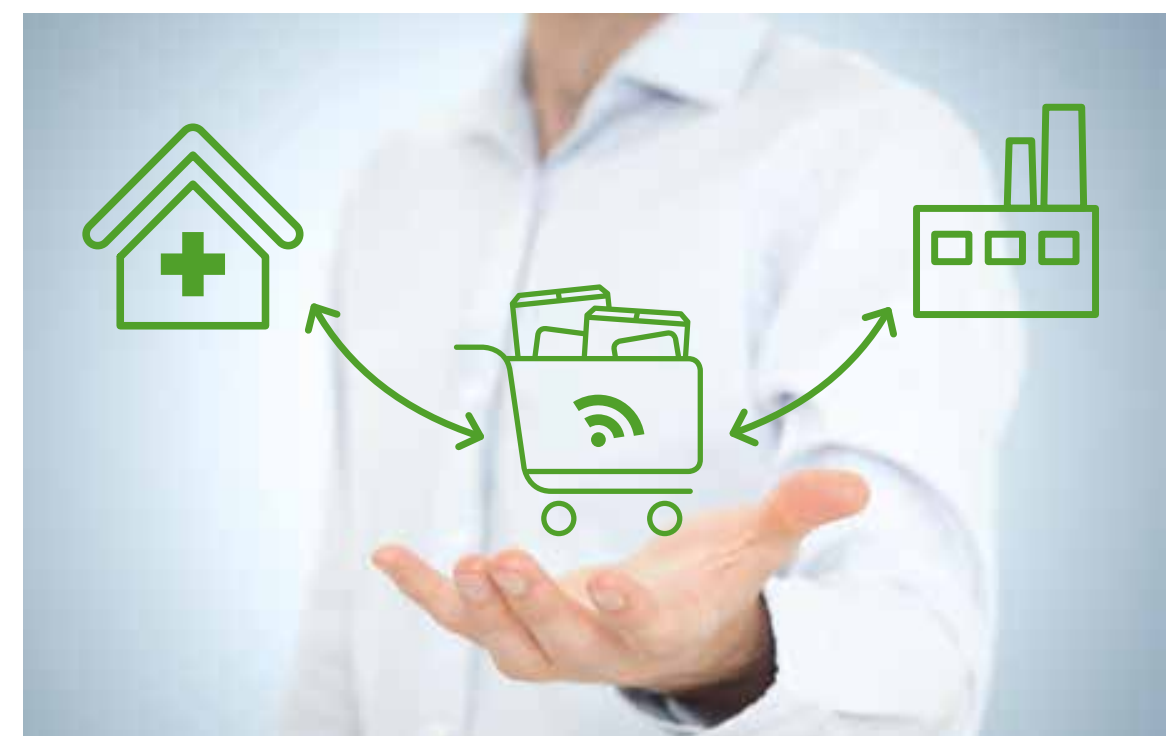

Symbolfoto: Klinik Einkauf Montage; AdobeStock / Jakub Jirsák

wendeten Produkte, von der Prothese bis hin zum Nahtmaterial, exakt zu erfassen, ist die Grundlage für eine genaue Deckungsbeitragsrechnung geschaffen. War diese genaue Zuordnung bis heute nur schwer darstellbar, bieten mittlerweile verschiedene Software-Lösungen eine Vielzahl an Möglichkeiten, die Transparenz in diesem Bereich zu erhöhen. Für die erfolgreiche Einführung und Umsetzung solcher Lösungen ist es zwingend erforderlich, Prozesse zu analysieren und diese zu verändern.

\section{Kommunikation ist Trumpf}

Die Rolle des Krankenhauseinkaufs hat sich entsprechend seiner stetig wachsenden Aufgaben in den letzten Jahren verändert, und wird dies auch zukünftig weiter tun. Als Bindeglied und Kommunikator zur Ge- schäftsführung, Projektverantwortlichen, vor allem aber auch zum medizinischem Fachpersonal nimmt er eine zentrale Rolle ein.

Die Notwendigkeit von Produktumstellungen, Lieferantenstraffungen oder Prozessharmonisierungen sind auf dem Papier schnell beschlossene Sache. Der Erfolg hängt aber nachhaltig von der frühen und guten Einbindung aller Betroffenen, vor allem der Anwender ab. Eine Kommunikation auf Augenhöhe bedeutet hierbei nicht, dass der Krankenhauseinkauf über die gleichen Fachkenntnisse verfügt wie der Oberarzt. Sie bedeutet vielmehr, dass im konstruktiven Austausch miteinander besprochen wird, welche wirtschaftlichen Auswirkungen die Konzentration auf ein 
bestimmtes Produkt hat, ohne dabei die klinischen Ergebnisse der Patientenversorgung negativ zu beeinflussen.

Doch nicht nur im Zusammenspiel mit anderen Abteilungen sind die kommunikativen Fähigkeiten des Krankenhauseinkaufs gefragt. Die Möglichkeiten, die vor allem die Digitalisierung von Prozessen in der Beschaffung bietet, werden nicht von jedem Mitarbeiter einer Einkaufsabteilung sofort erkannt. Ein gutes und effektives Change-Management ist notwendig, um optimierte Prozesse nachhaltig zu implementieren.

\section{Moderner Einkauf - moderne Einkaufs-} dienstleistung

Mit veränderten Anforderungen an den Krankenhauseinkauf müssen sich auch die Einkaufsdienstleister in Bezug auf ihr Leistungsspektrum weiterentwickeln. Das spiegelt sich auch in den Entscheidungsprozessen bei der Auswahl des Einkaufsdienstleisters wieder. War in der Vergangenheit ein guter Preis häufig das einzige Entscheidungskriterium für die Zusammenarbeit mit einem Einkaufsdienstleister, sind heute ergänzende Dienstleistungen wie Beratungsangebote im Rahmen der Prozessoptimierung, die Unterstützung bei der rechtssicheren Beschaffung sowie ein Angebot, das neben medizinischen Verbrauchsgütern und Investitionsgütern auch die Bedarfe in den Bereichen Pharmazie, Verpflegung sowie Software-Lösungen abdeckt, entscheidend.

Ein Einkaufsdienstleister als zentraler Ansprechpartner nicht nur für die Gesundheitseinrichtungen, sondern auch für die Vertragslieferanten bietet beiden Seiten entscheidende Vorteile, da er Markttrends wiederspiegeln, Bedürfnisse und Anforderungen beider Seiten kanalisieren und mehrwertstiftend für beide Seiten einsetzen kann. Die Erfahrung zeigt, dass die Zusammenarbeit mit Einkaufsdienstleistern, auf Krankenhausund Industrie-Seite, zur nachhaltigen Kostensenkung und einer deutlichen Effizienzsteigerung führt.
09 Der moderne Krankenhauseinkauf sieht sich neben vielen Chancen, die optimierte Prozesse und digitalisierte Arbeitsabläufe mit sich bringen, nach wie vor einigen Herausforderungen gegenübergestellt. So ist das Marktumfeld durch viele politische und regulative Initiativen, aber auch durch eine rechtlich teilweise unsichere Lage geprägt.

\section{Die Rolle des Kran- kenhauseinkaufs hat sich entspre- chend seiner ste- tig wachsenden Aufgaben in den letzten Jahren ver- ändert - und wird dies auch zukünf- tig weiter tun.}

Die Bedürfnisse aller, also auf der einen Seite kommunaler, konfessioneller und privater Krankenhäuser, über alle Versorgungsstufen hinweg, von mittelständischen Unternehmen bis hin zu internationalen Konzernen auf der anderen Seite in Einklang zu bringen, ist eine spannende Aufgabe. Durch den kontinuierlichen, intensiven Austausch mit allen Beteiligten lässt sich diese Aufgabe erfolgreich bewältigen und führt stets zum Erkenntnisgewinn für alle.
So ist beispielsweise eine frühzeitige $A b$ stimmung und Kommunikation zwischen Medizintechnikherstellern und Kliniken wichtig, um zu bewerten, ob und inwieweit das Inverkehrbringen von Medizinprodukten durch den Brexit für den deutschen Markt Auswirkungen hat. Nicht wenige aller in der EU-zertifizierten Medizinprodukte wurden durch benannte Stellen in Großbritannien zugelassen. Da diese Stellen nun wegfallen, gilt es, frühzeitig zu klären, ob die Versorgungssicherheit deutscher Krankenhäuser hiervon beeinflusst sein könnte. Um hier zeitnah an relevante Informationen zu kommen, hat beispielsweise die Prospitalia ihre 400 Lieferantenpartner stellvertretend für ihre 600 Vertragskrankenhäuser auf mögliche Auswirkungen des Brexits befragt. Die Ergebnisse wurden den Vertragseinrichtungen anschließend zentral zur Verfügung gestellt.

Die Förderung des Austauschs ist aber nicht die wichtigste Aufgabe von Einkaufsdienstleistern. Durch eine hohe Expertise hinsichtlich aller im Krankenhaus benötigten Produkte, v. a. medizinischer Güter, gelingt es ihnen in erster Linie, den Gesundheitseinrichtungen dabei zu helfen, eine hohe Qualität in der Gesundheitsversorgung und die Wirtschaftlichkeit der erbrachten Leistungen in Einklang zu bringen. Nicht zuletzt durch das Aufzeigen von Veränderungen auf der Erlösseite zur Rentabilitätsoptimierung auch im Bereich neuer Untersuchungs- und Behandlungsmethoden (NUB). Auf diese Weise tragen auch Einkaufsdienstleister ihren Teil zu einem effizienten, zukunftsfähigen Krankenhauseinkauf bei, der vor allem in der partnerschaftlichen Zusammenarbeit zwischen Gesundheitseinrichtung, Industrie und Einkaufsgemeinschaft nachhaltig zu erreichen ist.

\footnotetext{
Markus Wild ist CEO der Prospitalia, einer auf die Beschaffung von Medizinprodukten, Arznei- wie auch Lebensmitteln im Gesundheitswesen spezialisierten Unternehmensgruppe mit Tochtergesellschaften im In- und Ausland.
} 